\title{
Résumé de la surveillance des cas pédiatriques d'entérovirus D68 hospitalisés au Canada, septembre 2014
}

\author{
Edwin $\mathrm{JJ}^{1^{*}}$, Reyes Domingo $\mathrm{F}^{2}$, Booth $\mathrm{TF}^{3}$, Mersereau $\mathrm{T}^{2}$, Skowronski $\mathrm{DM}^{4}$, Chambers $\mathrm{C}^{4}$, Simmonds $\mathrm{K}^{5}$, \\ Scott $A N^{5}$, Winter $A L^{6}$, Peci $A^{6}$, Gubbay $J^{6}$, Drews $S J^{7}$, Krajden $M^{4}$, Karnauchow $T^{8}$, Smieja $M^{9}$, \\ Rempel $S^{1}$, Murti $M^{10}$, Pollock $S^{11}$, Gustafson $R^{12}$, Hoyano $D^{13}$, Allison $S^{14}$, Fathima $S^{7}$, Pabbaraju $K^{7}$, Wong \\ $\mathbf{S}^{7}$, Tellier $\mathbf{R}^{7}$, Tipples $\mathbf{G}^{7}$, Gad $\mathbf{R R}^{15}$, Mukhi $\mathrm{SN}^{3}$, Jafari $\mathbf{Y}^{16}$, Grudeski $E^{3}$, McDermid $A^{3}$ et Wong $\mathrm{T}^{2}$ \\ ${ }_{2}^{1}$ Centre pour l'infrastructure en santé publique, Agence de la santé publique du Canada, Ottawa (Ontario) \\ ${ }^{2}$ Centre de l'immunisation et des maladies respiratoires infectieuses, Agence de la santé publique du Canada, Ottawa \\ (Ontario) \\ ${ }^{3}$ Laboratoire national de microbiologie, Agence de la santé publique du Canada, Winnipeg (Manitoba) \\ ${ }^{4}$ Centre de contrôle des maladies de la Colombie-Britannique, Vancouver (Colombie-Britannique) \\ 5 Ministère de la Santé de l'Alberta, Edmonton (Alberta) \\ ${ }^{6}$ Santé publique Ontario, Toronto (Ontario) \\ ${ }^{7}$ Laboratoires provinciaux de santé publique de I'Alberta, Edmonton et Calgary (Alberta) \\ ${ }^{8}$ Centre hospitalier pour enfants de l'est de I'Ontario, Ottawa (Ontario) \\ ${ }^{9}$ Hamilton Regional Laboratory Medicine Program, Hamilton (Ontario) \\ ${ }^{10}$ Fraser Health Authority, Surrey (Colombie-Britannique) \\ ${ }^{11}$ Interior Health Authority, Kelowna (Colombie-Britannique) \\ ${ }^{12}$ Vancouver Coastal Health Authority, Vancouver (Colombie-Britannique) \\ ${ }^{13}$ Vancouver Island Health Authority, Victoria (Colombie-Britannique) \\ ${ }_{15}^{14}$ Northern Health Authority, Prince George (Colombie-Britannique) \\ ${ }_{16}^{15}$ Ministère de la Santé du Nouveau-Brunswick, Fredericton (Nouveau-Brunswick) \\ ${ }^{16}$ Ministère de la Santé et des Services sociaux des Territoires du Nord-Ouest, Yellowknife (Territoires du Nord-Ouest)
}

*Auteur-ressource : jonathan.edwin@phac-aspc.gc.ca

\section{Résumé}

Contexte : L'entérovirus D68 (EV-D68) est rarement détecté et n'a pas été associé à des cas graves au Canada. Au début de l'automne 2014, à la suite d'une augmentation inhabituelle de cas aux États-Unis, des grappes d'EVD68 chez des enfants et certains adultes montrant des symptômes graves ont été signalées au Canada.

Objectif : Fournir un résumé épidémiologique initial des cas pédiatriques hospitalisés avec l'EV-D68 au Canada.

Méthodologie : Projet pilote de surveillance d'une durée limitée visant à recueillir des renseignements sur les cas pédiatriques (enfants et adolescents de moins de 18 ans) d'EV-D68 hospitalisés entre le $1^{\text {er }}$ et le 30 septembre 2014 .

Résultats : Total de 268 cas signalés en Ontario $(n=210)$, en Alberta $(n=45)$ et en Colombie-Britannique $(n=$ 13). Parmi les 268 cas signalés, $64,9 \%(n=174)$ étaient des garçons; la différence relative au sexe était statistiquement significative $(p<0,01)$. L'âge a été signalé chez 255 cas, la moyenne d'âge chez les garçons étant de 5,4 ans et chez les filles de 5,3 ans. Parmi les cas ayant des données disponibles, 6,8 \% (18/266) ont été admis dans une unité de soins intensifs. Parmi les cas de maladie clinique documentée, 98,3\% (227/231) présentaient seulement une maladie respiratoire, $0,4 \%(n=1)$, seulement une maladie neurologique, et $0,9 \%$ $(n=2)$, les deux maladies; les cas sans maladie respiratoire ni neurologique étaient rares $(n=1)$. Parmi les 90 cas accompagnés d'informations cliniques supplémentaires, $43,3 \%$ présentaient de l'asthme. Aucun des 268 cas n'a entraîné le décès du patient.

Conclusion : L'éclosion de l'EV-D68 au Canada en septembre 2014 représente une nouvelle éclosion associée à une maladie grave chez les enfants. Ces observations constituent le premier résumé épidémiologique de cas graves liés à l'EV-D68, un agent pathogène respiratoire émergent au Canada. L'investigation continue de cet agent pathogène est nécessaire si l'on veut s'appuyer sur ces résultats et comprendre le spectre complet de sa maladie associée. 


\section{Introduction}

L'entérovirus D68 ou EV-D68 (genre entérovirus) est un entérovirus non poliomyélitique de la famille des Picornaviridae (1). Il fut découvert pour la première fois en 1962 après avoir été isolé chez quatre enfants malades présentant une pneumonie et une bronchiolite en Californie (2). Très peu de cas d'EV-D68 ont été détectés par le passé (3). De 1970 à 2005, le système de surveillance nationale des entérovirus des États-Unis n'a identifié que 26 cas d'EV-D68 parmi 49637 (0,1\%) échantillons d'entérovirus typés en laboratoire pendant cette période de 36 ans (4). Au Canada, un total de 82 cas d'EV-D68 ont été identifiés par le Laboratoire national de microbiologie de 1999 à 2013 (5).

Depuis 2008, plusieurs éclosions d'EV-D68 ont été signalées à l'échelle internationale, y compris aux Philippines (2008-2009) (6), au Japon (2010) (7, 8), aux Pays-Bas (2010) (9, 10), et aux États-Unis (2009-2010) (11, 12). La maladie rapportée variait de symptômes légers associés à une maladie respiratoire (fièvre, écoulement nasal, éternuements, toux et douleurs corporelles et musculaires) à une atteinte grave, la plupart du temps une pneumonie et une bronchiolite, nécessitant des soins intensifs (12). Des infections ont été signalées chez les enfants et chez les adultes; toutefois, les enfants âgés de moins d'un mois à quatre ans étaient le plus souvent touchés. Les enfants atteints d'une maladie respiratoire sous-jacente (p. ex. asthme, respiration sifflante et bronchiolite) et les adultes immunodéprimés étaient ceux le plus gravement touchés par la maladie $(13,14)$. L'épidémiologie et le spectre complet de la maladie causée par l'EV-D68 demeurent nébuleux $(12,13)$, principalement en raison de sa détection peu fréquente. Cependant, son émergence récente en tant qu'agent pathogène susceptible d'entraîner une épidémie et associé à une maladie grave (7-12) mérite une investigation.

\section{Description de l'événement}

À la mi-août 2014, les Centers for Disease Control and Prevention (CDC) des États-Unis ont été informés d'une augmentation de maladies respiratoires graves chez les enfants de Kansas City et de Chicago. Certains tests supplémentaires ont identifié l'EV-D68 comme l'agent étiologique prédominant parmi ceux détectés (13). De la mi-août au 11 décembre 2014, l'éclosion s'est propagée à travers tout le pays, entraînant 1149 cas d'EV-D68 confirmés en laboratoire et 12 décès, signalés dans 48 États en plus du District de Columbia (15). Les CDC examinent également depuis quelque temps des rapports d'enfants présentant une myélite flasque aiguë associée à une infection par l'EV-D68. Les investigations sont en cours afin de déterminer le rôle, le cas échéant, de l'infection par l'EV-D68 parmi les cas de maladie neurologique identifiés durant la même période $(15,16)$.

En septembre 2014, certaines provinces et territoires canadiens ont signalé des cas d'EV-D68 confirmés en laboratoire, certains étant associés à une atteinte neurologique $(17,18)$. En date du 15 décembre 2014, des cas d'EV-D68 confirmés en laboratoire, principalement chez les moins de 18 ans, ont été détectés dans les dix provinces et un territoire du Canada. L'EV-D68 ne figure pas parmi les maladies à déclaration obligatoire à l'échelle nationale au Canada, c'est pourquoi ses conséquences en tant que virus respiratoire saisonnier sont peu connues. Une sensibilisation accrue parmi le personnel de la santé publique et les cliniciens suivant les rapports de grappes aux États-Unis a probablement contribué à la détection des premiers cas au Canada.

En raison de l'émergence de tels cas au Canada et des connaissances limitées sur l'épidémiologie et le spectre complet de la maladie causée par l'EV-D68, l'Agence de la santé publique du Canada (ASPC), en collaboration avec divers organismes de santé publique provinciaux et territoriaux $(\mathrm{P} / \mathrm{T})$ participants, a mis en œuvre un projet pilote de surveillance des effets graves de l'EV-D68 d'une durée limitée en septembre 2014. Le but du présent article est de fournir un résumé épidémiologique des cas pédiatriques hospitalisés suivant l'émergence de l'éclosion de l'EV-D68 au Canada.

\section{Enquêtes}

En septembre 2014, toutes les agences de santé des provinces et territoires ont été invitées à participer au projet pilote de surveillance des effets graves de l'EV-D68. Les agences et centres de santé provinciaux et territoriaux suivants ont participé au projet pilote de surveillance : ministère de la Santé de l'Alberta; Centre de contrôle des maladies de la Colombie-Britannique (BCCDC); ministère de la Santé du Nouveau-Brunswick; ministère de la Santé et des Affaires sociales du Yukon; ministère de la Santé et des Services sociaux des Territoires du NordOuest; ministère de la Santé et des Services sociaux du Nunavut; Santé publique Ontario; Centre hospitalier pour 
enfants de l'est de l'Ontario; Hamilton Regional Laboratory Medicine Program (HRLMP) (desservant le Hamilton Health Sciences, St. Joseph's Healthcare Hamilton et autres hôpitaux communautaires du centre-sud de l'Ontario). La gestion du projet pilote et l'analyse épidémiologique ont été menées par le Centre de l'immunisation et des maladies respiratoires infectieuses de l'ASPC.

\section{Définition de cas}

Les cas étaient définis comme toutes personnes âgées de $\leq 18$ ans ayant été hospitalisées et ayant obtenu un résultat positif d'infection par l'EV-D68 au moyen du test par réaction de polymérisation en chaîne (PCR) et/ou du test de séquençage génétique du virus (par l'entremise de méthodes standard de génotypage des entérovirus) $(19,20)$, ou du test de PCR en temps réel spécifique à l'EV-D68 (21), à partir de tout échantillon clinique recueilli en septembre 2014.

\section{Collecte des données}

Les renseignements qui suivent étaient requis pour tous les cas, et ce, sous forme regroupée ou dans une liste détaillée anonyme : date de prélèvement de l'échantillon; sexe; âge; admission dans une unité de soins intensifs (USI); tableau clinique (p. ex. maladie respiratoire et/ou maladie neurologique); autre information clinique (asthme); et issue de la maladie ( $p$. ex. survie/décès du patient). La collecte des données était réalisée dans la mesure du possible à l'aide de formulaires de déclaration de cas de surveillance détaillés (disponibles sur demande).

\section{Analyse des données}

Des analyses descriptives de base ont été effectuées afin de générer un résumé épidémiologique des cas d'EVD68 hospitalisés. Les différences entre les groupes (garçons par rapport aux filles, et âge $<5$ ans par rapport à 5 à 18 ans) ont été soumises au test statistique du chi carré en supposant que l'EV-D68 touche un taux égal d'individus (par sexe et par âge) selon la distribution de la population dans les provinces/territoires déclarant les cas. Les valeurs attendues étaient calculées en utilisant les estimations de la population de 2014 de Statistique Canada (22).

\section{Évaluation}

Entre le $1^{\mathrm{er}}$ et le 30 septembre 2014,268 cas provenant de trois provinces ont été signalés : $210(78,4 \%)$ en Ontario (Santé publique Ontario, CHEO et HRLMP); 45 (16,8 \%) en Alberta; et 13 (4,9\%) en ColombieBritannique. Le Nouveau-Brunswick, les Territoires du Nord-Ouest, le Nunavut et le Yukon n'ont déclaré aucun cas avec date de prélèvement de l'échantillon durant le mois de septembre 2014. Fait à noter, les écoles primaires et secondaires publiques de la Colombie-Britannique étaient fermées durant ce mois en raison d'une grève des enseignants, ne reprenant leurs activités après les vacances de l'été que le 22 septembre 2014 (18). La collecte des données par le ministère de la Santé de l'Alberta et par le BCCDC a été effectuée au moyen de formulaires de déclaration de cas détaillés; par Santé publique Ontario, au moyen de réquisitions générales de test de laboratoire de Santé publique Ontario (23); et par le CHEO et le HRLMP, au moyen d'un examen des dossiers médicaux et de laboratoire. Près de la moitié de tous les cas ( 119 ou $44,4 \%$ ) présentaient des échantillons positifs d'EV-D68 recueillis durant la semaine 38 (14 au 20 septembre 2014), tandis que 60 cas $(22,4 \%)$ et 45 cas $(16,8 \%)$, respectivement, présentaient des échantillons positifs recueillis durant les semaines 39 (21 au 27 septembre) et 37 (7 au 13 septembre) (Figure 1). 
Figure 1 : Cas pédiatriques ( $\leq 18$ ans) d'EV-D68 hospitalisés, selon la semaine de prélèvement de l'échantillon; projet pilote de surveillance des effets graves de l'EV-D68, Canada, septembre 2014

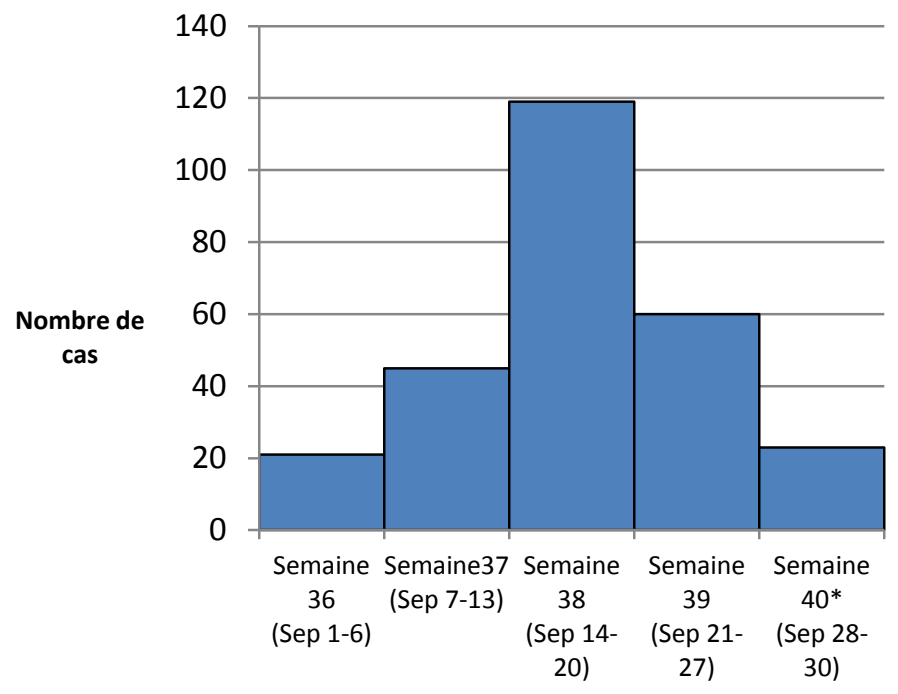

Semaine de prélèvement de l'échantillon

\footnotetext{
* Remarque : Les données de la semaine 40 ne sont pas complètes. En outre, cette courbe épidémiologique représente un aperçu limité dans le temps des cas pédiatriques hospitalisés en septembre 2014, et pourrait ne pas être représentative de l'ensemble de l'éclosion de l'EV-D68 au Canada (laquelle pourrait se poursuivre après cette période).
}

\section{Caractéristiques des cas}

Parmi les 268 cas dont la date de prélèvement de l'échantillon en septembre 2014 était connue, $174(64,9 \%)$ étaient des garçons; la différence à l'égard du sexe était statistiquement significative $(p<0,01)$ (Tableau 1). L'âge moyen des cas où l'âge était disponible $(n=255)$ était de 5,2 ans $(5,4$ ans chez les garçons et 5,3 ans chez les filles), tandis que l'âge médian était de 4,8 ans (5,0 ans chez les garçons et 5,0 ans chez les filles). La répartition des cas par groupes d'âge est présentée dans le tableau. La moitié des cas (50,6 \%) étaient des enfants âgés de moins de 5 ans et près du tiers des cas (35,7\%) étaient âgés entre 5 et 9 ans. La comparaison entre le nombre de cas observés chez les jeunes enfants ( $<5$ ans) et celui observé chez les 5 à 18 ans a montré une différence statistiquement significative $(p<0,01)$. Le nombre de cas observés chez les jeunes enfants $(n=129)$ était deux fois plus élevé que celui attendu d'après la distribution de la population. 
Tableau 1 : Résumé épidémiologique descriptif des cas pédiatriques d'EV-D68 hospitalisés; projet pilote de surveillance des effets graves de I'EV-68, Canada, septembre 2014

\begin{tabular}{|c|c|c|}
\hline $\begin{array}{l}\text { ( } N=\text { nombre de cas pour lesquels des } \\
\text { renseignements étaient disponibles) }\end{array}$ & $\mathbf{n}$ & $\%$ \\
\hline \multicolumn{3}{|l|}{ Sexe $(N=268)^{a}$} \\
\hline Garçons & 174 & 64.9 \\
\hline Filles & 94 & 35.1 \\
\hline \multicolumn{3}{|l|}{ Groupe d'âge (N = 255) } \\
\hline$<2$ ans & 56 & 22.0 \\
\hline $2-4$ ans & 73 & 28.6 \\
\hline $5-9$ ans & 91 & 35.7 \\
\hline $10-14$ ans & 31 & 12.1 \\
\hline $15-18$ ans & 4 & 1.6 \\
\hline \multicolumn{3}{|c|}{ Admission à l'unité des soins intensifs $(N=266)$} \\
\hline Oui & 18 & 6.8 \\
\hline Non & 248 & 93.2 \\
\hline \multicolumn{3}{|l|}{ Tableau clinique $(\mathbf{N}=\mathbf{2 3 1})$} \\
\hline Maladie respiratoire seulement & 227 & 98.3 \\
\hline Maladie neurologique seulement & 1 & 0.4 \\
\hline Maladie respiratoire et neurologique & 2 & 0.9 \\
\hline Aucune maladie respiratoire ni neurologique & 1 & 0.4 \\
\hline
\end{tabular}

Parmi les 266 cas dont le statut relatif à l'admission à l'USI était connu, 18 (6,8 \%) ont été admis dans une USI. Parmi ces 18 cas, $66,7 \%(n=12)$ étaient des garçons; la répartition selon le sexe était semblable à celle de l'ensemble des cas. Les âges moyen (5,5 ans) et médian (5,6 ans) des cas admis à l'USI étaient légèrement supérieurs à ceux de l'ensemble des cas. Comparativement aux âges moyen et médian de l'ensemble des cas, selon le sexe, ces valeurs étaient supérieures chez les garçons admis à l'USI (moyenne $=6,4$, médiane $=6,0$ ) et inférieures chez les filles admises à l'USI (moyenne $=4,0$, médiane $=3,6)$. Parmi les 231 cas accompagnés de données sur le tableau clinique, $229(99,1 \%)$ présentaient une maladie respiratoire et $3(1,3 \%)$ une maladie neurologique. Parmi ces trois derniers, un présentait des convulsions, un autre des symptômes de paralysie flasque aiguë, et un dernier des symptômes neurologiques non précisés. Un cas n'était associé à aucun symptôme respiratoire ni neurologique. Parmi les 90 cas accompagnés d'informations cliniques supplémentaires, 39 (43,3 \%) présentaient de l'asthme. Aucun des 268 cas n'a entraîné le décès du patient.

\section{Conclusion}

Aucune éclosion d'EV-D68 n'avait été détectée au Canada avant 2014. Les éclosions initiales d'agents pathogènes émergents sont habituellement d'abord reconnues grâce à leurs tableaux les plus graves, ce qui peut fausser la perception de la gravité globale de l'agent pathogène. La surveillance accrue au niveau de la 
population, qui inclut ultimement des formes plus légères de la maladie (même parmi les cas hospitalisés), élargit la compréhension du spectre de la maladie.

Même si une très grande proportion des cas pédiatriques des grappes initiales détectées dans les hôpitaux de Kansas City (100 \%) et de Chicago (91\%) en août 2014 ont nécessité des soins intensifs (13), cela ne représente en fait qu'un sous-groupe de tous les cas probablement survenus dans la région géographique desservie par ces hôpitaux. De même, notre surveillance accrue, bien que menée dans sept provinces et trois territoires, n'inclut que les cas d'EV-D68 confirmés en laboratoire ayant été hospitalisés. Lorsque nous examinons ces cas hospitalisés, l'âge médian calculé (4,8 ans) concorde avec celui observé initialement à Kansas City (4 ans) et à Chicago (5 ans) (13). La prépondérance importante de garçons que nous rapportons concorde également avec les autres résumés de surveillance de personnes infectées par un entérovirus ailleurs dans le monde (9). Comme c'est le cas avec les autres entérovirus, il est probable qu'une grande proportion des cas communautaires plus légers soient survenus sans que les patients ne cherchent à consulter ou qu'un test diagnostique soit nécessaire; ces tableaux légers et leurs caractéristiques épidémiologiques associées sont presque toujours omis dans la plupart des résumés de surveillance.

Bien que la majorité des cas observés depuis septembre 2014 aient été parmi les 18 ans et moins, un petit groupe de cas chez des adultes ont également été rapportés $(5,17,18)$. Toutefois, ces derniers ne sont pas inclus ici, car il a été décidé qu'aux fins d'aperçu précoce de l'éclosion et d'uniformité parmi les provinces et territoires participants, ce résumé épidémiologique se limiterait aux cas pédiatriques observés du $1^{\mathrm{er}}$ au 30 septembre 2014 seulement. La décision de nous concentrer sur les populations pédiatriques était appuyée par les données de plus en plus nombreuses démontrant que l'EV-D68 touche disproportionnellement les plus jeunes comparativement aux adultes (6-8, 10-13, 16, 24-27). D'autres cas ont été observés au Canada après le mois de septembre; par exemple, en Colombie-Britannique, où une grève des enseignants a retardé le retour automnal à l'école, un pic saisonnier subséquent d'EV-D68 a été observé. Par conséquent, il sera important de mettre en œuvre une surveillance continue des populations pédiatriques et adultes afin d'avoir une meilleure compréhension de la vulnérabilité liée à l'âge, et de la saisonnalité et de la gravité de la maladie.

Toutefois, il faut prendre garde de certaines limites associées à ces données. Ce résumé épidémiologique est axé sur une population spécifique (à risque élevé) et une période précoce de l'éclosion, et pourrait ne pas être représentatif de l'ensemble de l'éclosion de l'EV-D68 au Canada en 2014. En outre, parmi les cas pour lesquels une admission à l'USI a été signalée, on ne sait pas dans quelle mesure l'infection à l'EV-D68 a contribué aux admissions à l'USI. De plus, les provinces et les territoires ont mené des tests de laboratoire pour l'EV-D68 au moyen de différentes méthodes qui, de par leur nature, présentent divers degrés de sensibilité et de spécificité $(19,28)$. Étant donné que l'EV-D68 n'est pas une maladie à déclaration obligatoire ni à l'échelle nationale ni à l'échelle provinciale (et territoriale), certaines provinces (et territoires) ont fait face à divers obstacles au début du suivi accru et du signalement des cas, utilisant des approches différentes dans la collecte des données. Ceci a probablement causé la perte de cas au suivi et pourrait expliquer une portion des résultats manquants dans ce rapport. De même, les provinces (et territoires) ne s'équivalent pas toutes pour ce qui est de leur capacité d'intervention devant des événements de santé publique émergents et imprévus. L'expérience avec l'EV-D68 sert de rappel au fait que si l'on veut de bonnes interventions de santé publique en temps opportun, cela nécessite des lois et une infrastructure de soutien permettant l'investigation des agents pathogènes émergents qui, de par leur nature même, apparaissent sans préavis et ne figurent pas nécessairement dans les listes de maladies à déclaration obligatoire.

Le projet pilote de surveillance des effets graves de l'EV-D68 représente la première tentative de description des caractéristiques épidémiologiques des cas pédiatriques graves d'EV-D68 à l'échelle nationale au Canada, et fournit un aperçu précieux des premiers stades de l'éclosion de 2014. Les limites liées à ces observations devraient être prises en compte au moment d'extrapoler l'expérience à l'ensemble du Canada ou de faire des comparaisons entre les provinces ou territoires. Une investigation continue est requise afin de mieux définir la saisonnalité, le degré de vulnérabilité des patients et le spectre de la maladie associés à cet agent pathogène émergent, et de mettre en œuvre des mesures appropriées de santé publique afin d'en atténuer les répercussions. 


\section{Remerciements}

La rédaction de ce rapport n'aurait pas été possible sans la contribution de tous les organismes et centres de santé fédéraux, provinciaux et territoriaux qui ont participé ou ont fourni des données au projet pilote canadien de surveillance des effets graves de l'EV-D68. En outre, nous aimerions remercier les personnes suivantes : $D^{\text {re }}$ Dana Paquette, directrice du Programme canadien d'épidémiologie de terrain; $D^{r}$ Robert Stirling, médecin en santé publique, Santé publique Ontario; le personnel des laboratoires provinciaux de santé publique de l'Alberta pour leur analyse systématique des échantillons; la province de la Colombie-Britannique aimerait remercier les infirmières et épidémiologistes de la santé publique des autorités sanitaires régionales qui ont rempli les formulaires de déclaration de cas et qui ont compilé les renseignements de la surveillance accrue, avec des remerciements particuliers à Christina Fung, Margot Smythe, Maritia Gully, Kelly Yu et Gillian Frosst; les personnes suivantes du ministère de la Santé du Nouveau-Brunswick (Shelley Landsburg, Suzanne Savoie et Louis-Alexandre Jalbert); Réseau de santé Vitalité et la collaboration spéciale du laboratoire de microbiologie du centre hospitalier universitaire George L. Dumont; Réseau de santé Horizon; ministère de la Santé et des Affaires sociales du Yukon; bureau de l'administrateur en chef de la santé publique des Territoires du Nord-Ouest; ministère de la Santé du gouvernement du Nunavut; Emily De Rubeis et Veeran-Anne Singh de la Direction générale de la santé des Premières nations et des Inuits de Santé Canada.

\section{Conflit d'intérêts}

Aucun

\section{Financement}

Aucun

\section{Références}

(1) International Committee on Taxonomy of Viruses, King AMQ. Virus taxonomy: Classification and nomenclature of viruses: Ninth report of the international committee on taxonomy of viruses. London; Waltham, MA: Academic Press; 2012:1327.

(2) Schieble $\mathrm{JH}$, Fox VL, Lennette EH. A probable new human picornavirus associated with respiratory diseases. Am J Epidemiol. 1967;85(2):297-310.

(3) Oberste MS, Maher K, Schnurr D, et al. Enterovirus 68 is associated with respiratory illness and shares biological features with both the enteroviruses and the rhinoviruses. J Gen Virol. 2004; 85(Pt 9):2577-84. doi: 10.1099/vir.0.79925-0.

(4) Khetsuriani N, Lamonte-Fowlkes A, Oberst S, Pallansch MA, Centers for Disease Control and Prevention. Enterovirus surveillance-United States, 1970-2005. MMWR Surveill Summ. 2006; 55(8):1-20. doi: ss5508a1.

(5) National Collaborating Centre for Infectious Diseases. Disease debrief: EV-D68. Updated 2014 [cited 2014 Dec]. http://www.nccid.ca/disease-debrief-ev-d68.

(6) Imamura T, Fuji N, Suzuki A, et al. Enterovirus 68 among children with severe acute respiratory infection, the Philippines. Emerg Infect Dis. 2011; 17(8):1430-5. doi: 10.3201/eid1708.101328.

(7) Kaida A, Kubo H, Sekiguchi J, et al. Enterovirus 68 in children with acute respiratory tract infections, Osaka, Japan. Emerg Infect Dis. 2011; 17(8):1494-7. doi: 10.3201/eid1708.110028.

(8) Ikeda T, Mizuta K, Abiko C, et al. Acute respiratory infections due to enterovirus 68 in Yamagata, Japan between 2005 and 2010. Microbiol Immunol. 2012;56(2):139-43. doi: 10.1111/j.1348-0421.2012.00411.x.

(9) Meijer A, Benschop KS, Donker GA, van der Avoort HG. Continued seasonal circulation of enterovirus D68 in the Netherlands, 2011-2014. Eurosurveillance. 2014; 19(42). http://www.eurosurveillance.org/ViewArticle.aspx?Articleld=20935

(10) Meijer A, van der Sanden S, Snijders BE, et al. Emergence and epidemic occurrence of enterovirus 68 respiratory infections in the Netherlands in 2010. Virology. 2012; 423(1):49-57. doi: 10.1016/j.virol.2011.11.021.

(11) Jacobson LM, Redd JT, Schneider E, et al. Outbreak of lower respiratory tract illness associated with human enterovirus 68 among American Indian children. Pediatr Infect Dis J. 2012;31(3):309-12. doi: 10.1097/INF.0b013e3182443eaf.

(12) Centers for Disease Control and Prevention (CDC). Clusters of acute respiratory illness associated with human enterovirus 68-Asia, Europe, and United States, 2008-2010. MMWR. 2011; 60(38):1301-4. doi: mm6038a1.

(13) Midgley CM, Midgley CM, Selvarangan R, et al. Severe respiratory illness associated with enterovirus D68-Missouri and Illinois, 2014. MMWR. 2014; 63(36):798-9. 
(14) Hasegawa S, Hirano R, Okamoto-Nakagawa R, Ichiyama T, Shirabe K. Enterovirus 68 infection in children with asthma attacks: Virus-induced asthma in Japanese children. Allergy. 2011; 66(12):1618-20. doi: 10.1111/j.13989995.2011.02725.x.

(15) Human enterovirus D68 North America (20): Update. ProMED. 2014 Dec 15. Archive No. 20141215.3035008. http://www.promedmail.org/direct.php?id=3035008

(16) Pastula DM, Aliabadi N, Haynes AK, et al. Acute neurologic illness of unknown etiology in children-Colorado, August-September 2014. MMWR. 2014; 63(40):901-2. doi: mm6340a5.

(17) Fonseca K, Kellner JD., Talbot J, et al. Human enterovirus 68-Canada: (Alberta). ProMED. 2014 Sep 16 [cited 2014 Dec]. Archive No. 20140916.2775534. http://www.promedmail.org/direct.php?id=2775534

(18) Murti M, Pollock S, Krajden M, Tang P, Chambers C, Skowronski D. Polio-like illness-North America: Canada (British Columbia). 2014 Sep 30 [cited 2014 Dec 29]. http://www.promedmail.org/direct.php?id=2819618

(19) Nix WA, Oberste MS, Pallansch MA. Sensitive, seminested PCR amplification of VP1 sequences for direct identification of all enterovirus serotypes from original clinical specimens. J Clin Microbiol. 2006;44(8):2698-704. doi: 44/8/2698.

(20) Rahamat-Langendoen J, Riezebos-Brilman A, Borger R, et al. Upsurge of human enterovirus 68 infections in patients with severe respiratory tract infections. J Clin Virol. 2011; 52(2):103-6. doi: 10.1016/j.jcv.2011.06.019.

(21) Centers for Disease Control and Prevention (CDC). Enterovirus D68 (EV-D68) 2014 outbreak strain-specific real-time reverse transcription / polymerase chain reaction (rRT-PCR) assay instructions-Version 10/14/2014 [cited 2014 Dec 30]. http://www.cdc.gov/non-polio-enterovirus/downloads/EV-D68-RT-PCR-protocol.pdf

(22) Statistics Canada. Table 051-0001-Estimates of population, by age group and sex for July 1, Canada, Provinces and Territories, annual (persons unless otherwise noted). CANSIM. (disponible en français: http://www5.statcan.gc.ca/cansim/a26?id=510001\&retrLang=fra\&lang=fra)

(23) Public Health Ontario. General test requisition [cited 2014 Dec 23]. http://www.publichealthontario.ca/en/eRepository/General_test_fillable_requisition.pdf

(24) Enterovirus targets children in central United States. Medical Lāboratory Observer. 2014; 46(10):6.

(25) Baum SG. Severe respiratory illness associated with EV-D68. NEJM Journal Watch. Infectious Diseases. 2014:n/a. doi: http://dx.doi.org/10.1056/nejm-jw.NA35680.

(26) Dietz V, Andrus J, Olive JM, Cochi S, de Quadros C. Epidemiology and clinical characteristics of acute flaccid paralysis associated with non-polio enterovirus isolation: The experience in the Americas. Bull World Health Organ. 1995; 73(5):597-603.

(27) Renois F, Bouin A, Andreoletti L. Enterovirus 68 in pediatric patients hospitalized for acute airway diseases. J Clin Microbiol. 2013; 51(2):640-3. doi: 10.1128/JCM.02640-12.

(28) Oberste MS, Penaranda S, Rogers SL, Henderson E, Nix WA. Comparative evaluation of taqman real-time PCR and semi-nested VP1 PCR for detection of enteroviruses in clinical specimens. J Clin Virol. 2010;49(1):73-74. doi: 10.1016/j.jcv.2010.06.022. 